\title{
A Routing Scheme for a New Irregular Baseline Multistage Interconnection Network
}

\author{
Mamta Ghai \\ Department of Computer Science \& Engineering \\ Punjabi University \\ Patiala( India)
}

\begin{abstract}
Parallel processing is an efficient form of information processing system, which emphasizes the exploitation of concurrent events in the computing process. To achieve parallel processing it's required to develop more capable and costeffective systems. In order to operate more efficiently a network is required to handle large amount of traffic. Multi-stage Interconnection Network plays a vital role on the performance of these multiprocessor systems. In this paper an attempt has been made to analyze the characteristics of new class of Irregular Fault-Tolerant Multistage Interconnection network named as Irregular Modified Baseline Multistage Interconnection network IMABN and an efficient routing procedure has been defined to study the fault-tolerance of the network. Fault-Tolerance in an interconnection network is very important for its continuous operation over a relatively long period of time. Fault-Tolerance is an ability of the network to operate in presence of multiple faults. The behavior of the Proposed IMABN has been analyzed and compared with regular network MABN under fault free conditions and in presence of faults. In IMABN there are six possible paths between source and destinations whereas MABN has only four. Thus the proposed IMABN is more Fault-tolerant than existing regular Modified Augmented Baseline multistage interconnection network (MABN).
\end{abstract}

Keywords- Multistage Interconnection network; Fault-Tolerance; Augmented Baseline Network.

\section{INTRODUCTION}

With the present state of technology building multiprocessor system with hundreds of processors is feasible. A vital component of these systems is the interconnection network(IN) that enables the processors to communicate among themselves or with the memory units. Multipath nature of multistage interconnection networks become more popular. Many ways of providing fault-tolerance to multistage interconnection networks(MINs) have been proposed. The basic idea for fault-tolerance is to provide multiple paths between source-destination pair so that alternate paths can been used in case of faults. Sufficient work has been done on the regular type of MINs, but little attention has been paid to the irregular type of MIN.

In this paper, a new class of irregular Baseline multistage interconnection network named as irregular modified augmented baseline network(IMABN) is proposed. In this paper we present methods of increasing fault-tolerance of an network by introducing the extra stage. Hence with the additional stage more paths available between each source and destination, as compared to existing network MABN. The proposed Irregular Modified Augmented Baseline Network(IMABN) is an Modified augmented baseline network(MABN) with additional stage. In an IMABN, there are Six possible paths between any source-destination pair, whereas MABN has only Four. Fault-Tolerance in an Interconnection network is very important for its continuous operation over a relatively long period of time. It is the ability of the network to operate in presence of multiple faults, although at a degraded performance. There are many ways to increase the fault-tolerance of the network.

(1) Increasing the no of stages.

(2) Providing multiple links between stages.

(3) Increasing size of switch.

(4) Incorporating multiple copies of a basic network.

This paper has been organized into five sections whose details are as follows:

Section I introduces the subject under study. Section II describe the structure and design of the networks. Section III focus on the routing tags. Section IV describe the routing procedure for the proposed IMABN.Finally, some concluding remarks are given in section $\mathrm{V}$.

\section{StRUCTURE OF NETWORKS}

\section{A. MABN (Modified Augmented Baseline Network)}

To construct an MABN of size $\mathrm{N}$, two identical groups of N/2 sources and N/2 destinations need to be formed first. Each source is linked to both the groups via multiplexers. There is one $4 \times 1$ MUX for each input link of a switch in stage 1 and one 1 x 4 DEMUX for each output link of a switch in stage n2. MABN consists of two identical sub-networks which are denoted by $\mathrm{G}^{\mathrm{i}}$. Switches A, B, C, D belonging to stage 1 of a sub-network $\left(\mathrm{G}^{\mathrm{i}}\right)$ form a conjugate subset,switches $A$ and $B$ form a conjugate pair, and switches $\mathrm{A}$ and $\mathrm{C}$ form a conjugate loop. An MABN of size 16X16 is shown in Figure 1. 


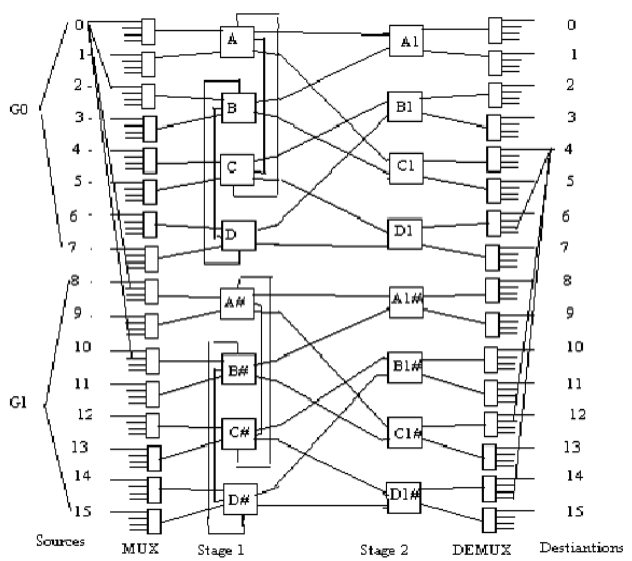

Figure 1: An MABN of size 16 X 16.

\section{B. IMABN( Irregular MABN)}

IMABN (Irregular modified augmented baseline network) is a Modied Augmented Baseline Network with one additional stage, increase size of switch and more auxiliary links. To construct an IMABN of size $\mathrm{N}$ i.e. $\mathrm{N}$ sources and $\mathrm{N}$ destinations, two identical groups of N/2 sources and N/2 destinations need to be formed first. Each source is linked to both the groups via multiplexers. There is one $4 \times 1$ MUX for each input link of a switch in stage 1 and one 1 x 4 DEMUX for each output link of a switch in stage n-1. IMABN consists of two identical sub-networks which are denoted by $G^{i}$. For example, in Figure 2, switches A, B, C, D belonging to stage 1 of a subnetwork $\left(G^{i}\right)$ form a conjugate subset, switches $A$ and $\mathrm{B}$ form a conjugate pair, and switches $\mathrm{A}$ and $\mathrm{C}$ form a conjugate loop.Thus an IMABN of size $\mathrm{N}$ consists of $\mathrm{N}$ number of 4 x 1 MUXs, $N$ number of 1 x 4 DEMUXs, and switches in the last stage of size $2 \times 2$, switches in the middle stage of size $5 \times 5$ and switches in the first stage of size $3 \times 3$. IMABN as its name suggest is an irregular network in which middle (additional) stage doesn't have equal number of switches as of other stages. The irregular topology of IMABN varies the number of switching elements encountered in the way of data transfer through an input-output pair depending on the path chosen, which makes the average rate of failure of the network less as compared to that of regular $\mathrm{ABN}$ and MABN.IMABN is a dynamically re-routable irregular MIN and provides multiple paths of varying lengths between a source-destination pair.

Observe that this construction procedure has two benefits. First, the network can tolerate the failure of any switch in the network. And, secondly it provides a topology which lends itself to on-line repair and maintainability, as a loop can be removed from any stage of the IMABN without disrupting the operation of the network. Since the sub-networks are identical, so the VLSI implementation of the network becomes simple. IMABN of size $16 \times 16$ is shown in Fig 2.

\section{ROUTING TAGS}

A source selects a particular subnetwork $\left(\mathrm{G}^{\mathrm{i}}\right)$ based upon the most significant bit of the destination. each source is connected to two switches (primary and secondary) in a subnetwork.

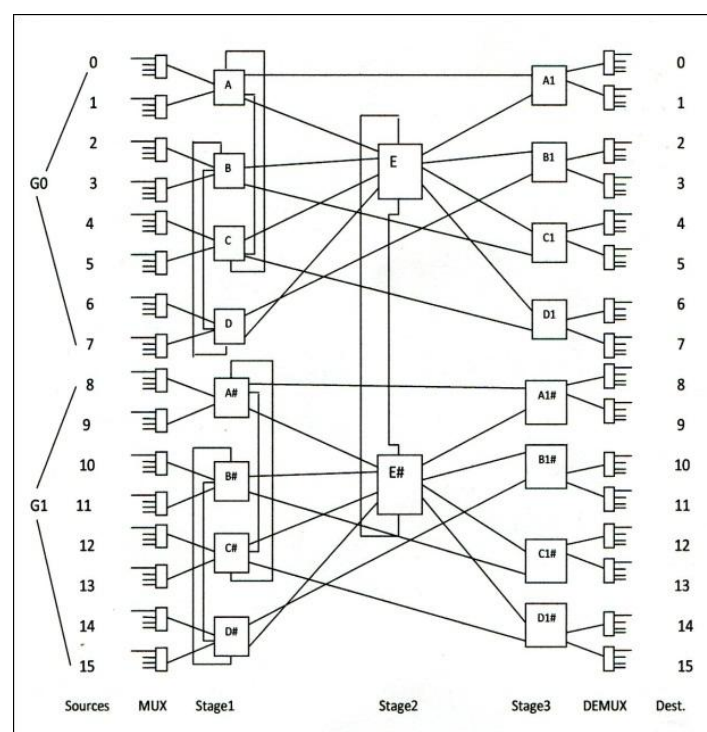

Figure 2: An IMABN of size 16 X 16

Let the source $\mathrm{S}$ and destination $\mathrm{D}$ be represented in binary code as:

$$
\begin{aligned}
& \mathrm{S}=\mathrm{s}_{0}, \mathrm{~s}_{1}, \ldots, \mathrm{s}_{\mathrm{n}-2}, \mathrm{~s}_{\mathrm{n}-1} \\
& \mathrm{D}=\mathrm{d}_{0}, \mathrm{~d}_{1}, \ldots, \mathrm{d}_{\mathrm{n}-2}, \mathrm{~d}_{\mathrm{n}-1}
\end{aligned}
$$

(i) Source $\mathrm{S}$ is connected to the $\left(\mathrm{s}_{1}, \ldots, \mathrm{s}_{\mathrm{n}-2}\right)$ primary switch in both the sub-networks through the multiplexers.

(ii) Source $\mathrm{S}$ is also connected to the $\left[\left\{\left(\mathrm{s}_{1}, \ldots, \mathrm{s}_{\mathrm{n}-2}\right)+1\right\} \bmod \right.$ N/4] secondary switch in both the sub-networks through the multiplexers.

\section{ROUTING PROCEDURE}

Routing procedure for a network tells that how a request from any source $S$ travels through the various intermediate stages and reaches to a given destination D. Following assumptions have been made for the algorithm.

- Each source destination pair tries to utilize only one path at a time.

- Source \& switches have the ability to detect faults in the switches to which they are connected.

\section{A. Routing Scheme for IMABN(Irregular MABN)}

A request from any source $S$ to a given destination $D$ is routed through the IMABN as:

1) For each source: The source $\mathrm{S}$ selects one of the subnetwork $\mathrm{G}^{\mathrm{i}}$ based on the most significant bit of the destination $\mathrm{D}\left(\mathrm{i}=\mathrm{d}_{0}\right)$. Each source attempts entry into the IMABN via its primary path. If the primary path is faulty, then the request is routed to secondary path. If the secondary path is also faulty then the request is routed to the other subnetwork of the IMABN, via auxiliary links of stage 2 . If still request doesn't get matured, then the request is rerouted to the secondary subnetwork, in which same routing is followed as in the case of primary sub-network.

2) For each switch in stage $n$ - 3: After the MUX, the routing of the request in the first (n-3) stage of the sub- 
network depends upon one tag bit, which depends on $\mathrm{d}_{1} \mathrm{~d}_{2}$ destination address bits. Routing tag bit for stage 1 is calculated as follows :

If $\mathrm{d}_{1} \mathrm{~d}_{2}=00$,

then both conjugate pairs in the sub-network will have tag bit $=0$.

If $\mathrm{d}_{1} \mathrm{~d}_{2}=01$,

then first conjugate pair(A/A\#, B/B\#) will have tag bit $=1$, and Second conjugate pair $(\mathrm{C} / \mathrm{C} \#, \mathrm{D} / \mathrm{D \#})$ will have tag bit $=0$.

If $\mathrm{d}_{1} \mathrm{~d}_{2}=10$,

then both conjugate pairs in the sub-network will have tag bit $=1$.

If $\mathrm{d}_{1} \mathrm{~d}_{2}=11$,

then first conjugate pair(A/A\#, B/B\#) will have tag bit $=0$, and Second conjugate pair $(\mathrm{C} / \mathrm{C} \#, \mathrm{D} / \mathrm{D \#})$ will have tag bit $=1$.

3) For each switch in stage $n$ - 2: For a request at a switch in stage $n-2$, value of tag bit is given as below:

If $\mathrm{d}_{1} \mathrm{~d}_{2}=00$,

then at $\mathrm{E}$,if request comes from(A/A\#,B/B\#) then it goes to Node A1( use tag bit 00 ) and if request comes from (C/C\#,D/D\#) then it goes to node B1( use tag bit 01).

If $\mathrm{d}_{1} \mathrm{~d}_{2}=01$,

then at $\mathrm{E}$, if request comes from $(\mathrm{A} / \mathrm{A \#}, \mathrm{B} / \mathrm{B} \#)$ then it goes to Node $\mathrm{C} 1$ (use tag bit 10) and if request comes from $(\mathrm{C} / \mathrm{C} \#, \mathrm{D} / \mathrm{D} \#)$ then it goes to node B1(use tag bit 01)

If $\mathrm{d}_{1} \mathrm{~d}_{2}=10$,

then at $\mathrm{E}$,if request comes from(A/A\#,B/B\#) then it goes to Node $\mathrm{C} 1$ (use tag bit 10) and if request comes from $(\mathrm{C} / \mathrm{C} \#, \mathrm{D} / \mathrm{D} \#)$ then it goes to node D1(use tag bit 11)

If $\mathrm{d}_{1} \mathrm{~d}_{2}=11$,

then at $\mathrm{E}$,if request comes from(A/A\#,B/B\#) then it goes to Node A1(use tag bit 00) and if request comes from $(\mathrm{C} / \mathrm{C} \#, \mathrm{D} / \mathrm{D} \#)$ then it goes to node D1(use tag bit 11)

4) For each switch in stage $n-1$ : For a request at a switch in stage $n-1$, use bit $d_{n-1}$ of the routing tag and route the request accordingly to one of the output links. If the required output link is busy, then repeat step two and three in the secondary sub-network. If again the required output link is busy in stage $\mathrm{n}-2$, then drop the request.

5) For each demultiplexer at the output of stage $n-1$ : For routing a request through a DEMUX, following concept is used.

If destination and MUX are in same sub-network, then $1^{\text {st }}$ MUX uses output line 00 and $2^{\text {nd }}$ MUX uses output line 10.

If destination and MUX are in different subnetworks, then $1^{\text {st }}$ MUX uses output line 01 and $2^{\text {nd }}$ MUX uses output line 11.

Multiple paths between $\mathrm{S}=0000$ and $\mathrm{D}=0100$ of an IMABN are shown below.

Case 1:Routing in IMABN

Primary Path:

$$
\begin{aligned}
& \text { 0-> } \operatorname{MUX}(0)-\mathrm{A}-\mathrm{E}-\mathrm{C} 1-\operatorname{DEMUX}(4)-4 \\
& 0->\operatorname{MUX}(0)-\mathrm{A}-\mathrm{C}-\mathrm{D} 1-\operatorname{DEMUX}(6)-4
\end{aligned}
$$

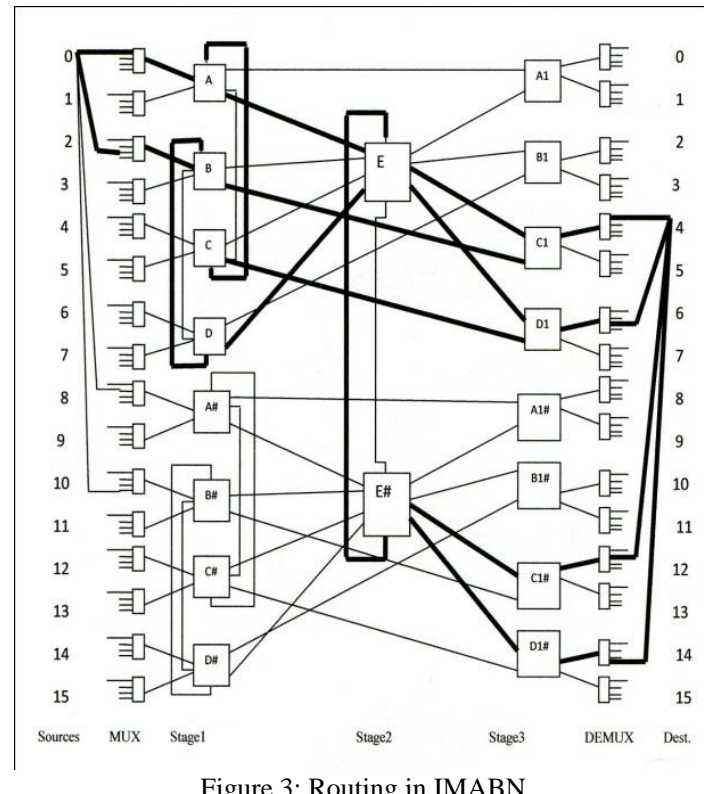

Figure 3: Routing in IMABN

Primary path using auxiliary links of stage 2:-

$$
\text { 0-> } \operatorname{MUX}(0)-\mathrm{A}-\mathrm{E} \text { - E\# - C1\# - DEMUX(12) - } 4
$$

Secondary path:

0-> MUX(2) - B - C1 - DEMUX(4) - 4

0-> $\operatorname{MUX}(2)-\mathrm{B}-\mathrm{D}-\mathrm{E}-\mathrm{D} 1-\operatorname{DEMUX}(6)-4$

Secondary path using auxiliary links of stage 2:-

0-> MUX(2) - B - D - E - E\# - D1\# - DEMUX(14) - 4 
Case 2: Routing in IMABN

Primary\# Path:

$$
\begin{aligned}
& \text { 0->MUX(8) - A\# - E\# - C1\# - DEMUX(12) - } 4 \\
& \text { 0->MUX(8) - A\# - C\# - D1\# - DEMUX(14) - } 4
\end{aligned}
$$

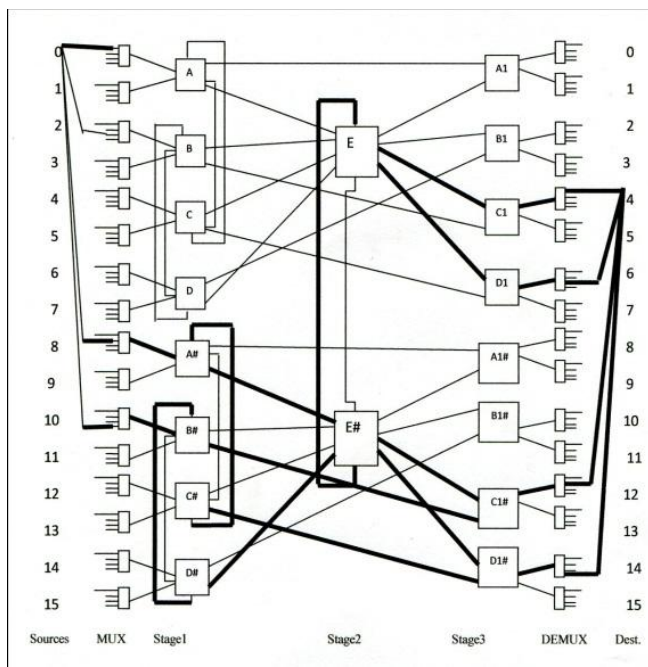

Figure 4: Routing in IMABN

Primary \# path using auxiliary links of stage2:-

0->MUX(8) - A\# - E\# - E -C1 - DEMUX(4) - 4

Secondary \# path:

0->MUX(10) - B\# - C1\# - DEMUX(12)

0->MUX(10) -B\# - D\# - E\# - D1\# - DEMUX(14) - 4

Secondary \# path using auxiliary links of stage2:-

0->MUX(10) - B\# -D\# - E\# - E - D1 - DEMUX(6) - 4

\section{CONCLUSIONS}

An Irregular Modified Augmented Baseline Network (IMABN) is designed from regular Modified Augmented Baseline network (MABN).IMABN is dynamically re-routable and providing multiple paths of varying lengths between source and destination pairs. It has been found that IMABN has six possible paths whereas in MABN has only four. Thus IMABN is more fault-tolerant than MABN.

\section{REFERENCES}

[1] Bhuyan Laxmi N., Yang Qing and Aggarwal P. Dharma, "Performance of Multiprocessor Interconnection Networks", Proceeding of IEEE, February 1989, pp. 25-37.

[2] Bhogavilli Suresh K. and Abu-Amara Hosame, "Design and Analysis of High Performance Multistage Interconnection Networks", IEEE Transactions on Computers, vol. 46, no. 1, January 1997, pp. 110 -117.

[3] Bansal P.K, Singh Kuldeep and Joshi R.C, " On Fault tolerant Multistage Interconnection Network", Conference on Computer Electrical Engineering, vol. 20, no.4, 1994, pp. 335-345.

[4] Blaket James T. and Trivedi Kishor S., "Reliabilities of Two FaultTolerant Interconnection Networks", Proceeding of IEEE, 1988, pp. 300-305.

[5] Charles Chenggong Charles and Bruck Jehoshua, "Tolerating Multiple Faults in Multistage Interconnection Networks with Minimal Extra Stages", IEEE Transactions on Computers, vol. 49, no. 9, September 2000, pp. 998-1004.

[6] Cheema Karamjit, Aggarwal Rinkle, “Design Scheme and Performance Evaluation of new Fault-Tolerant Multistage Interconnection Network”, IJCSNS International Jounal of Computer Science and Network Security , VOL.9 NO.9,September 2009.

[7] Mamta Ghai et al., " Performance Analysis of Fault-Tolerant Irregular baseline multistage interconnection network", IJCSE International journal on computer science \& engineering Vol. 02, No. 09, 2010, 30793084

[8] Mamta Ghai et al., “ Design and Reliability Analysis of New FaultTolerant Irregular Multistage Interconnection network", IJCA International Journal of Computer Applications(0975-8887) Volume 10, No 1, November 2010.

[9] Nitin, "On Analytic Bounds of Regular and Irregular Fault-tolerant Multi-stage Interconnection Networks”, Proceedings of International Conference, 2006.

[10] Sadawarti Harsh and Bansal P.K., “ Fault Tolerant Irregular Augmented Shuffle Network", Proceeding of the 2007 WSEAS International Conference on Computer Engineering and Applications, Australia, January 17-19,2007. pp. 7-12.

[11] Sengupta J. and Bansal P.K, "Performance of Regular and Irregular Dynamic MINs", Proceeding of International Conference IEEE TENCON, 1999, pp. 427-430.

[12] Sharma Sandeep and Bansal P.K., "A New Fault Tolerant Multistage Interconnection Network", Proceeding of International Conference IEEE TENCON, 2002, pp. 347-350.

[13] Rao, D. S. (2010). Performance Evaluation of Node Failure Prediction QoS Routing Protocol ( NFPQR ) in Ad Hoc Networks. International Journal of Advanced Computer Science and Applications - IJACSA, $1(6), 54-59$.

[14] A Survey on Attacks and Defense Metrics of Routing Mechanism in Mobile Ad hoc Networks. International Journal of Advanced Computer Science and Applications - IJACSA, 2(3), 7-12.

[15] Beebi, P. K. A. (2011). A Study on Cross Layer MAC design for performance optimization of routing protocols in MANETs. International Journal of Advanced Computer Science and Applications IJACSA, 2(2), 11-16

[16] Suri, P. K. (2011). Simulation of Packet Telephony in Mobile Adhoc Networks Using Network Simulator. International Journal of Advanced Computer Science and Applications - IJACSA, 2(1), 87-92. 